\title{
Dynamics of turbulence under the effect of stratification and internal waves
}

\author{
O. A. Druzhinin ${ }^{1}$ and L. A. Ostrovsky ${ }^{2}$ \\ ${ }^{1}$ The Institute of Applied Physics, Russian Acad. Sci., Nizhny Novgorod, Russia \\ ${ }^{2}$ NOAA Environmental Science Research Lab., Boulder, CO, USA \\ Correspondence to: O. A. Druzhinin (druzhinin@hydro.appl.sci-nnov.ru)
}

Received: 29 December 2014 - Published in Nonlin. Processes Geophys. Discuss.: 19 February 2015

Revised: 27 April 2015 - Accepted: 12 May 2015 - Published: 4 June 2015

\begin{abstract}
The objective of this paper is to study the dynamics of small-scale turbulence near a pycnocline, both in the free regime and under the action of an internal gravity wave (IW) propagating along a pycnocline, using direct numerical simulation (DNS). Turbulence is initially induced in a horizontal layer at some distance above the pycnocline. The velocity and density fields of IWs propagating in the pycnocline are also prescribed as an initial condition. The IW wavelength is considered to be larger by the order of magnitude as compared to the initial turbulence integral length scale. Stratification in the pycnocline is considered to be sufficiently strong so that the effects of turbulent mixing remain negligible. The dynamics of turbulence is studied both with and without an initially induced IW. The DNS results show that, in the absence of an IW, turbulence decays, but its decay rate is reduced in the vicinity of the pycnocline, where stratification effects are significant. In this case, at sufficiently late times, most of the turbulent energy is located in a layer close to the pycnocline center. Here, turbulent eddies are collapsed in the vertical direction and acquire the "pancake" shape. IW modifies turbulence dynamics, in that the turbulence kinetic energy (TKE) is significantly enhanced as compared to the TKE in the absence of IW. As in the case without IW, most of the turbulent energy is localized in the vicinity of the pycnocline center. Here, the TKE spectrum is considerably enhanced in the entire wave-number range as compared to the TKE spectrum in the absence of IW.
\end{abstract}

\section{Introduction}

Interaction between small-scale turbulence and internal gravity waves (IWs) plays an important role in the processes of mixing that have a direct impact on the dynamics of the seasonal pycnocline in the ocean (Phillips, 1977; Fernando, 1991). Turbulence in the mixed region above the pycnocline can be produced by breaking surface waves driven by the wind forcing or due to shear-flow instabilities. In laboratory studies of the effect of small-scale turbulence on the pycnocline, in the absence of mean shear, turbulent motions are usually induced by an oscillating grid (Turner, 1973; Thorpe, 2007).

One of the most interesting and practically important aspects of the turbulence-IW interaction in the absence of mean shear is the effect of damping of IWs by turbulence on the one hand, and the possibility of enhancement of smallscale turbulence by non-breaking IWs on the other hand. The phenomenon of IW damping by turbulence was observed in early laboratory experiments by Phillips (1977) and Kantha (1980). Quantitative measurements of the IW damping effect were first performed in a laboratory experiment by Ostrovsky et al. (1996). In the latter experiment, IWs were generated in the pycnocline by a wave maker, and small-scale turbulence was induced by an oscillating grid at some level above the pycnocline. Measurements and comparison of the IW amplitudes, with and without turbulence, showed an effective enhancement of the decay rate of IW under the effect of turbulence, which was found to be in good agreement with the theoretical prediction by Ostrovsky and Zaborskikh (1996) based on a semi-empirical closure approach. 
The effect of damping of IWs propagating through a pycnocline by a forced turbulent layer above the pycnocline was recently studied with the use of direct numerical simulation (DNS) by Druzhinin et al. (2013). The results show that if the ratio of IW amplitude to turbulent pulsations amplitude is sufficiently small (less than 0.5), turbulence strongly enhances IW damping. In this case, the damping rate obtained in DNS agrees well with the prediction of a semi-empirical closure approach developed by Ostrovsky and Zaborskikh (1996). However, for larger IW amplitudes, the effect of damping of IWs by turbulence is much weaker, and, in this case, the semi-empirical theory overestimates the IW damping rate by the order of magnitude.

The effect of enhancement of small-scale turbulence by mechanically generated, non-breaking internal waves was experimentally observed by Matusov et al. (1989). In that experiment, an oscillating grid was used to induce turbulence above a pycnocline. An internal gravity wave propagating in the pycnocline was generated by a wave maker. The results of this experiment show that a sufficiently strong (as compared to turbulent grid-induced velocity fluctuations), nonbreaking internal wave can significantly increase the kinetic energy of turbulence in the well-mixed layer above the pycnocline. However, the obtained experimental data did not provide enough detail to study the modification of turbulence kinetic energy spectra under the influence of IWs.

A somewhat similar phenomenon has been experimentally observed in the ocean boundary layer where the kinetic energy of turbulence and its dissipation rate can be significantly enhanced in the presence of surface gravity waves relative to the wind-stress production (cf., e.g., Anis and Moum, 1995). The results of recent numerical simulations by Tsai et al. (2015) also show that non-breaking surface waves can effectively increase turbulence kinetic energy in the vicinity of the water surface.

The objective of the present paper is to study the possibility of the enhancement of small-scale turbulence by an internal gravity wave (IW) propagating in a pycnocline, and to consider the case where the initial IW amplitude is on the order of, or larger than, the turbulent pulsation amplitude. The results of DNS by Druzhinin et al. (2013) show that, under this condition, the damping of IW by turbulence remains negligible. As in the latter study, the buoyancy jump across the pycnocline is considered to be large enough, such that the effects related to turbulent mixing remain insignificant. The main focus here is to investigate in more detail the effect of the enhancement of small-scale turbulence by an IW propagating in the pycnocline, and, in particular, to separate the contributions of the IW velocity field and the turbulent velocity field produced by the IW in the fluid kinetic energy spectrum.

The setup and parameters of the numerical experiment are discussed in Sect. 2. Initialization of internal waves and their properties are discussed in Sect. 3. The dynamics of turbulence in the absence of the initially excited IW is discussed

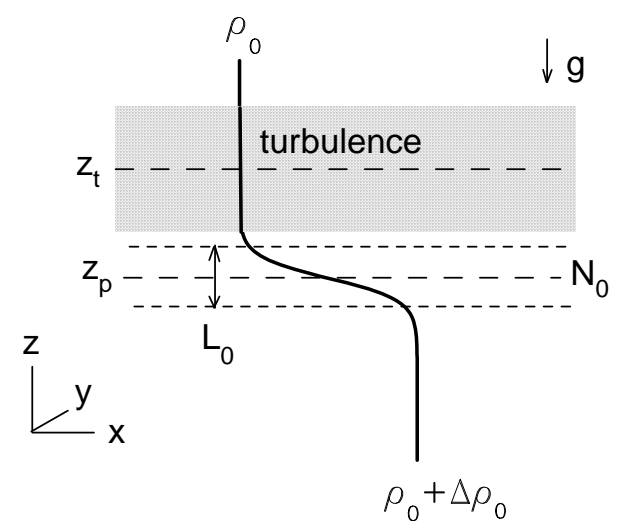

Figure 1. Schematic of the numerical experiment: $x, y$ and $z$ are the Cartesian coordinates; $\rho_{0}$ is the density above the pycnocline; $\Delta \rho_{0}$ the density jump across the pycnocline; $g$ the gravity acceleration, $N_{0}$ the buoyancy frequency in the pycnocline center; $L_{0}$ the pycnocline thickness; $z_{\mathrm{p}}$ and $z_{\mathrm{t}}$ the locations of the pycnocline and the turbulent layer centers.

in Sect. 4. The effect of an IW propagating in the pycnocline on turbulence dynamics is discussed in Sect. 5. Conclusions and discussion of numerical results and estimates of the enhancement of small-scale turbulence by IWs in laboratory and natural conditions are presented in Sect. 6.

\section{Numerical method and initial conditions}

We consider a stably stratified fluid with a pycnocline (Fig. 1). The initial turbulence field is localized in a layer at some distance above the pycnocline. The first mode of the internal wave propagating along the pycnocline from left to right is also prescribed as an initial condition. Periodic boundary conditions in the horizontal, $x$ and $y$, directions and a Neumann (zero normal gradient) boundary condition in the vertical $z$ direction are considered. The thickness of the pycnocline, $L_{0}$, and the buoyancy frequency in the middle of the pycnocline, $N_{0}=\left(-\frac{g}{\rho_{0}} \frac{\mathrm{d} \rho_{0}}{\mathrm{~d} z}\right)^{1 / 2}$ (where $g$ is the gravity acceleration and $\rho_{0}(z)$ the fluid density), are chosen to define the characteristic length and timescales, $L_{0}$ and $T_{0}=1 / N_{0}$, which are used further to write the governing equations in the dimensionless form.

The Navier-Stokes equations for the fluid velocity are written under the Boussinesq approximation as (Phillips, 1977)

$\frac{\partial U_{i}}{\partial t}+U_{j} \frac{\partial U_{i}}{\partial x_{j}}=-\frac{\partial P}{\partial x_{i}}+\frac{1}{R e} \frac{\partial^{2} U_{i}}{\partial x_{j}^{2}}-R i \delta_{i z} \rho$,

$\frac{\partial U_{j}}{\partial x_{j}}=0$.

The equation for the fluid density is 


$$
\frac{\partial \rho}{\partial t}+U_{j} \frac{\partial \rho}{\partial x_{j}}-U_{z} N_{\text {ref }}^{2}(z)=\frac{1}{\operatorname{RePr}} \frac{\partial^{2} \rho}{\partial x_{j}^{2}} .
$$

In Eqs. (1)-(3), $U_{i}(i=x, y, z)$ is the instantaneous fluid velocity, and $\rho$ and $P$ are the instantaneous deviations of the fluid density and pressure from the respective hydrostatic profiles; $x_{i}=x, y, z$ are the Cartesian coordinates.

Reynolds and Richardson numbers are defined as

$$
R e=\frac{U_{0} L_{0}}{v}, R i=\left(\frac{L_{0} N_{0}}{U_{0}}\right)^{2} .
$$

The Prandtl number is $P r=v / \kappa$, where $v$ is the fluid kinematic viscosity and $\kappa$ the molecular diffusivity. The coordinates, time and velocity are normalized by the length scales, timescales and velocity scales, $L_{0}, T_{0}$ and $U_{0}=L_{0} / T_{0}$. Note that, since the timescale is defined as $T_{0}=1 / N_{0}$ and the velocity scale as $U_{0}=L_{0} / T_{0}=L_{0} N_{0}$, the Richardson number in DNS identically equals unity, $R i=1$. The density deviation $\rho$ is normalized by the density jump across the pycnocline, $\Delta \rho_{0}$ (Fig. 1).

The dimensionless reference profile of the buoyancy frequency, $N_{\text {ref }}(z)$, is prescribed in the form

$N_{\text {ref }}(z)=\frac{1}{\cosh 2\left(z-z_{\mathrm{p}}\right)}$,

where $z_{\mathrm{p}}$ defines the pycnocline location, and the dimensionless reference density profile, $\rho_{\text {ref }}(z)$, is

$$
\begin{aligned}
\rho_{\mathrm{ref}}(z) & =\rho_{\mathrm{ref}}(-\infty)-\int_{-\infty}^{z} N_{\mathrm{ref}}^{2}(z) \mathrm{d} z=\rho_{\mathrm{ref}}(-\infty) \\
& -0.5 \tanh 2\left(z-z_{\mathrm{p}}\right),
\end{aligned}
$$

where $\rho_{\text {ref }}(-\infty)$ can be an arbitrary constant since its value does not influence the integration of Eqs. (1)-(3). Thus, for convenience, $\rho_{\text {ref }}(-\infty)$, is set equal to 1.5 , and the reference density profile is rewritten in the form

$\rho_{\mathrm{ref}}(z)=1+0.5\left[1-\tanh 2\left(z-z_{\mathrm{p}}\right)\right]$.

The dimensionless instantaneous density is obtained as a sum of $\rho_{\text {ref }}(z)$ and the instantaneous density deviation, $\rho$. Note that the dimensional density can be obtained as a sum $\left[\rho_{0}+\Delta \rho_{0}\left(0.5\left[1-\tanh 2\left(z-z_{p}\right)\right]+\rho\right)\right]$ where $\rho_{0}$ is the dimensional reference (undisturbed) density above the pycnocline.

The Navier-Stokes equations for the fluid velocity and density Eqs. (1)-(3) are integrated into a cubic domain with sizes $0 \leq x \leq 40,-10 \leq y \leq 10$ and $0 \leq z \leq 20$ by employing a finite difference method of second-order accuracy on a uniform rectangular staggered grid consisting of $400 \times 200 \times$ 200 nodes in the $x, y$ and $z$ directions, respectively. The integration is advanced in time using the Adams-Bashforth method with time step $\Delta t=0.01$. The Poisson equation for the pressure is solved by FFT transform over the $x$ and $y$ coordinates, and the Gaussian elimination method over the $z$ coordinate (Druzhinin et al., 2013). The Neumann (zero normal gradient) boundary condition is prescribed for all fields in the horizontal $(x, y)$ planes at $z=0$ and $z=20$, and periodic boundary conditions are prescribed in the longitudinal $(x)$ and transverse $(y)$ directions.

In DNS, we prescribe the Reynolds number to be $R e=20000$. This number is sufficiently large to render the viscous damping of IWs negligible. The Prandtl number $\mathrm{Pr}$ is set equal to unity.

\section{Internal waves}

The initial condition for the velocity and density fields is prescribed as a first mode of internal wave field with wavelength $\lambda$ (and wave number $k=2 \pi / \lambda$ ) and frequency $\omega$. The solution of the linearized Eqs. (1)-(3) for the progressive internal wave propagating from left to right in the $x$ direction can be defined as (Phillips, 1977)

$U_{x}^{I W}(x, z, t)=-\frac{1}{k} \frac{\mathrm{d} W(z)}{\mathrm{d} z} \sin (k x-\omega t)$,

$U_{z}^{I W}(x, z, t)=W(z) \cos (k x-\omega t)$,

$\rho_{z}^{I W}(x, z, t)=\frac{W(z)}{\omega} \frac{\mathrm{d} \rho_{\mathrm{ref}}}{\mathrm{d} z} \sin (k x-\omega t)$.

The initial conditions for the IW field are taken from Eqs. (8)-(10) at $t=0$. Function $W(z)$ is obtained as an eigenfunction of the well-known Taylor-Goldstein boundary problem (Phillips, 1977):

$\frac{d^{2} W}{\mathrm{~d} z^{2}}+\left(\frac{N^{2}}{\omega^{2}}-1\right) k^{2} W=0$,

with conditions $W(z) \rightarrow W_{0} \exp \left[k\left(z-z_{\mathrm{p}}\right)\right]$ for $z \ll z_{\mathrm{p}}$, and $W(z) \rightarrow W_{0} \exp \left[-k\left(z-z_{\mathrm{p}}\right)\right]$ for $z \gg z_{\mathrm{p}}$, where $W_{0}$ is the IW velocity amplitude at $z=z_{\mathrm{p}}$. The problem Eq. (11) was solved by the shooting method with matching at the pycnocline center, $z=z_{\mathrm{p}}$ (Hazel, 1972). The distribution of the first-mode vertical velocity in the IW and the dispersion relation $\omega(k)$ for wave numbers in the range $0.3<k<6$ obtained numerically for wavelength $\lambda=10$ are presented in Fig. 2a. The figure shows that, as expected, the energy of the first mode is concentrated around the pycnocline.

DNS was performed with initial conditions Eqs. (8)-(10) at $t=0$ corresponding to the IW fields with wavelength $\lambda=$ 10 (frequency $\omega=0.489$, period $T \approx 13$ ). Previous DNS results by Druzhinin et al. (2013) show that weak IWs of short length (say, about 3 times smaller as compared to the $\lambda=10$ considered in the present paper) are severely damped by turbulence. The results show that the damping rate of IWs with the amplitude 2 times less than the turbulence amplitude grows as $1 / \lambda^{2}$. On the other hand, if we consider larger 
(a)
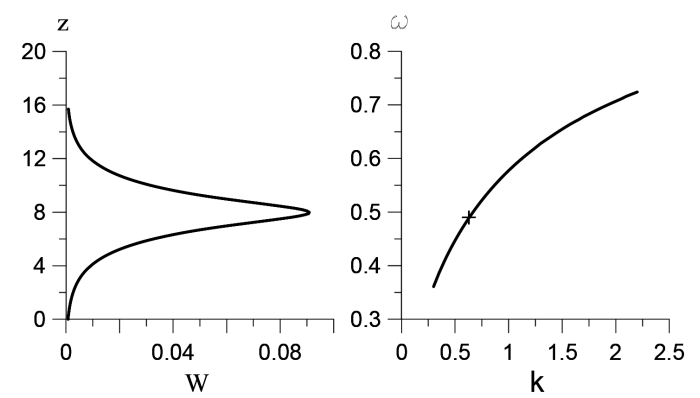

(b)
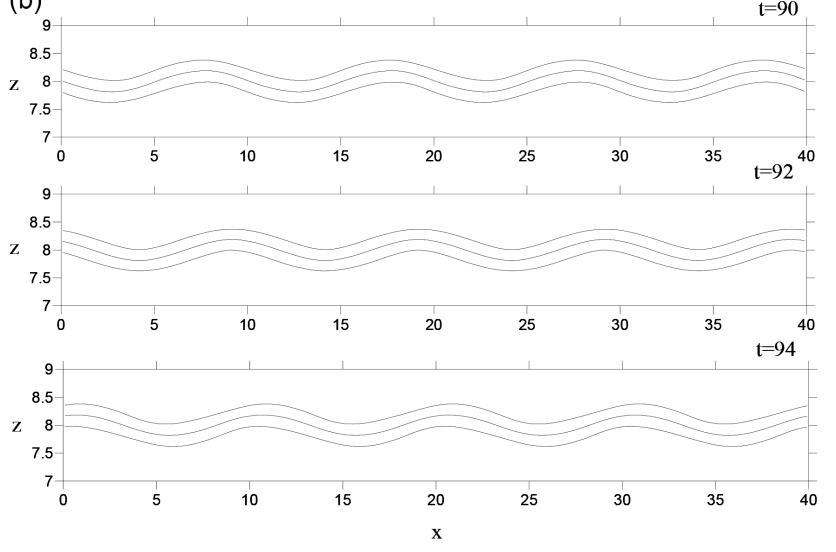

Figure 2. (a) Distribution of the vertical velocity $W(z)$ for wavelength $\lambda=10$ (left) and the dispersion relation $\omega(k)$ (right) for the first IW mode. The wave number of the selected wavelength is shown by a symbol. (b) The instantaneous contours of the density deviation obtained in the central $(x, z)$ plane at different time moments in DNS with initial condition (Eqs. 5-7) prescribed for IW, propagating from left to right with wavelength $\lambda=10$ (frequency $\omega=0.489$, phase velocity $c \approx 0.78$ ) and amplitude $W_{0}=$ 0.1 . There is no initially induced turbulence. Density contours are 1.3, 1.5 and 1.7. Contour 1.5 marks the location of the pycnocline center.

IW amplitudes and reduce the IW length, the wave slope increases so that strong, short-length IWs become strongly nonlinear and are prone to breaking and viscous dissipation. In the present study, the IW amplitude was prescribed as $W_{0}=0.1$. Figure $2 \mathrm{~b}$ shows isopycnal displacements $\mathrm{ob}-$ tained in DNS at different times with initial conditions prescribed for an IW with selected wavelength. In this case, the amplitude of the isopycnal displacement is about $a \approx 0.2$, and the wave slope is about $k a=2 \pi a / \lambda \approx 0.12$, which may be regarded as small enough to ensure that nonlinear effects during the IW propagation in the pycnocline remain negligible. Below (in Fig. 7), spatial IW spectra also show that amplitudes of higher harmonics remain negligible as compared to the first-harmonics amplitude.

\section{Dynamics of turbulence in the absence of IW}

In order to investigate how turbulence evolves in the absence of internal waves, DNS was performed with the initially induced turbulence field and no imposed IW field. The midpycnocline level was prescribed at $z_{\mathrm{p}}=8$ and the turbulence layer center was set at $z_{\mathrm{t}}=10$. The values of $z_{\mathrm{p}}$ and $z_{\mathrm{t}}$ were chosen to ensure that the effects of turbulent mixing and internal wave generation by turbulence in the pycnocline remained sufficiently small for the considered initial amplitude of turbulent velocity (defined below).

The turbulent velocity field is initialized in DNS as a random, divergence-free field in the form

$\left.U_{i}(x, y, z)=U_{\mathrm{t} 0} U_{i}^{f}(x, y, z) \exp \left[-0.5\left(z-z_{\mathrm{t}}\right)^{2}\right)\right]$,

where $i=x, y, z \cdot U_{i}^{f}(x, y, z)$ is a homogeneous isotropic field with a given power spectrum in the form

$E(k)=E_{0} k \exp \left(-\frac{k}{k_{f}}\right)$,

where wave number $k_{f}$ defines the spectral location of the energy peak. Factor $E_{0}$ is chosen so that the amplitude (i.e., an absolute maximum value of the modulus of $\left.U_{i}^{f}(x, y, z)\right)$ equals unity. Thus, parameter $U_{\mathrm{t} 0}$ in Eq. (12) defines the turbulence velocity amplitude; $U_{\mathrm{t} 0}$ and $k_{f}$ were prescribed in DNS as $U_{\mathrm{t} 0}=0.1$ and $k_{f}=1$. Numerical results show that, in this case, the effects of turbulent mixing on the pycnocline structure and generation of internal waves by turbulence remain negligible during the considered time interval $(0<t<400$ in dimensionless units). For the considered choice of the spectrum given by Eq. (13) with $k_{\mathrm{f}}=1$ the turbulence dimensionless integral length scale, $L_{\mathrm{t}}$, at initialization is on order unity. Thus, the turbulent Reynolds number, $R e_{\mathrm{t}}$, based on $L_{\mathrm{t}}$ and $U_{\mathrm{t} 0}$, is evaluated as $R e_{\mathrm{t}}=L_{\mathrm{t}} U_{\mathrm{t} 0} R e \approx$ 2000.

The mean vertical profiles of the velocity and density fields, $\left\langle U_{i}\right\rangle(z)$ and $<\rho>(z)$, were obtained by averaging over the horizontal $(x, y)$ plane performed for each $z$. Root mean square (rms) deviations of the velocity and density were then obtained as

$$
\begin{aligned}
U_{i}^{\prime} & =\left(<U_{i}^{2}-<U_{i}>^{2}>\right)^{1 / 2}, \rho^{\prime} \\
& =\left(<\rho^{2}-<\rho>^{2}>\right)^{1 / 2} .
\end{aligned}
$$

The vertical mean profile of the mean kinetic energy, $E(z)$, was evaluated as

$E=\frac{1}{2} \sum_{i=x, y, z} U_{i}^{\prime 2}$

Another important characteristic of the dynamics of turbulence in a density stratified fluid is the gradient Richardson 
number, $R i_{g}$ (Phillips, 1977). In the considered case, there is no mean shear. Thus, the gradient Richardson number parameter can be evaluated via the mean buoyancy frequency, $N$, and the mean turbulent shear stress defined by the TKE dissipation rate and kinematic viscosity, $(\varepsilon / \nu \equiv \varepsilon R e)$ (Thorpe, 2007), as

$R i_{g}=-\frac{R i}{\varepsilon R e} \frac{d<\rho>}{\mathrm{d} z}=\frac{N^{2}}{\varepsilon R e}$,

where the dissipation rate, $\varepsilon$, can be obtained from DNS data by averaging over the $(x, y)$ plane for each $z$ in the form (Phillips, 1977)

$\varepsilon=\frac{1}{R e} \sum_{i}<\left(\frac{\partial \widetilde{U}_{i}}{\partial x}\right)^{2}+\left(\frac{\partial \widetilde{U}_{i}}{\partial y}\right)^{2}+\left(\frac{\partial \widetilde{U}_{i}}{\partial z}\right)^{2}>$

In Eq. (17), $\widetilde{U}_{i}=U_{i}-<U_{i}>, i=x, y, z$ is the instantaneous deviation of the velocity from the mean value.

Figure 3a shows vertical profiles of different rms velocity components, $U_{x}^{\prime}, U_{y}^{\prime}$ and $U_{z}^{\prime}$, and mean density, $\left.<\rho\right\rangle$ (left panel), and the Richardson number, $R i_{g}$ (right panel), obtained in DNS at time moments $t=100$ and $t=400$ with no initially induced IW. (Here and below in Fig. $4 \mathrm{a}$, the $R i_{g}(z)$ profile is cut off at the level of unity for $R i_{g}(z)>1$.) The figure also shows the density reference (initial) profile, $\rho_{\text {ref }}(z)$, and the profiles of the rms velocity components $U_{x}^{\prime}$ and $U_{z}^{\prime}$ of the internal wave without an initially induced turbulence layer. The figure shows that $x$ and $y$ rms velocities coincide in the region sufficiently far from the pycnocline (for $z>11$ ). In this region, the gradient Richardson number remains sufficiently small $\left(R i_{g}<0.2\right.$ at $\left.t=400\right)$, so that it can be regarded as weakly stratified. On the other hand, in the region $z<10$, sufficiently close to the pycnocline, $R i_{g}>1$ and vertical rms velocity, $U_{z}^{\prime}$, are much smaller as compared to the horizontal rms velocities, $U_{x}^{\prime}$ and $U_{z}^{\prime}$, whose amplitudes peak at level $z=9$ at $t=400$. Thus, in this strongly stratified region, turbulent motion becomes quasi-two-dimensional and there is a collapse of three-dimensional vortices and the formation of pancake eddies (cf. Fig. 3e below). Figure 3a also shows that the mean density profile, $\langle\rho\rangle(z)$, practically coincides with the reference profile, $\rho_{\text {ref }}(z)$, during the considered time interval. That means that the effect of turbulent mixing on the pycnocline structure remains negligible.

Figure $3 \mathrm{a}$ also shows that vertical rms velocity increases in the vicinity of the pycnocline center (at $z=8$ ) where $U_{x}^{\prime}, U_{y}^{\prime}, U_{z}^{\prime}$ are on the same order. This increase can be attributed to the presence of internal waves excited by decaying turbulence in the pycnocline. The presence of these turbulence-generated IWs is confirmed by numerical results in Fig. 3b. The figure shows temporal development of the density at the point with coordinates $x=20, y=10$ and $z=8$ (i.e., at the pycnocline center in the middle of the computational domain). The figure also shows the temporal development of the density in an initially induced internal wave without initially excited turbulence for comparison. The figure shows that small, finite density variations are present in the pycnocline, which can be attributed to weak internal waves excited by turbulence. The analysis of the frequency spectrum of the density oscillations in the pycnocline and the structure of isopycnals (not presented here) shows that mostly first- and second-mode IWs are generated by turbulence with corresponding frequencies $\omega_{1} \approx 0.8$ and $\omega_{2} \approx 0.2$ and wavelength $\lambda_{\mathrm{t}} \approx 4$. (More details about the physical mechanism responsible for the IW generation by turbulence in a pycnocline are provided, e.g., by Kantha, 1979, and Carruthers and Hunt, 1986). The amplitude of these turbulencegenerated IWs remains smaller by the order of magnitude as compared to the amplitude of the IW induced in the pycnocline due to the initial condition (Eqs. 8-10).

Figure $3 \mathrm{c}$ shows the temporal development of $x, y$ and $z$ rms velocity components, $U_{x}^{\prime}, U_{y}^{\prime}, U_{z}^{\prime}$, obtained in DNS at different $z$ levels $(z=9,10$ and 11). The figure shows that turbulence decays, and the decay rate is different at different $z$ levels. At $z=11$, the rms velocities remain on the same order at all times. At levels $z=10$ and $z=9$, component $U_{z}^{\prime}$ diminishes at a greater rate as compared to the $x$ and $y$ components, $U_{x}^{\prime}$ and $U_{y}^{\prime}$, so that at sufficiently late times $(t>200$ for $z=10$ and $t>50$ at $z=9$ ), vertical velocity becomes smaller almost by the order of magnitude as compared to the $x$ - and $y$-velocity components. That means that in the region sufficiently close to the pycnocline, there is a collapse of three-dimensional turbulence under the effect of stable stratification and fluid motion becomes quasi-two-dimensional.

Temporal development of the mean kinetic energy, $E$, at different $z$ levels $(z=9,10$ and 11), is presented in Fig. 3d. The figure shows that $E$ decays at a lower rate in the region in the vicinity of the pycnocline (at $z=9$ ) as compared to levels $z=10$ and $z=11$ where stratification is weak. The figure shows that $E(z=11) \sim t^{-1.6}$, whereas $E(z=9) \sim t^{-0.9}$ at times $t>50$.

In the experimental study of strongly stratified homogeneous decaying grid turbulence, Praud et al. (2005) observed that kinetic energy decays as $t^{-1.3}$, which is close to the decay law of non-stratified grid turbulence (Warhaft and Lumley, 1978). Praud et al. (2005) also observed formation of pancake vortex structures at sufficiently late times. In the present study, the initial turbulence distribution is inhomogeneous in the $z$ direction, so that TKE dynamics at a given location is governed not only by viscous dissipation, but also by turbulent diffusion of momentum. Therefore, the reduction of turbulence kinetic energy is also modified by turbulent momentum transport due to the inhomogeneity of turbulence. A reduced decay rate observed in our DNS in the strongly stratified region $\left(t^{-0.9}\right.$ at $\left.z=9\right)$ as compared to the decay rate $t^{-1.6}$ in the region with weaker stratification (at $z=11$ ) can be attributed to the growth of the horizontal scale of turbulence due to the development of quasi-two-dimensional, pancake vortex structures (to be discussed below). 
(a)

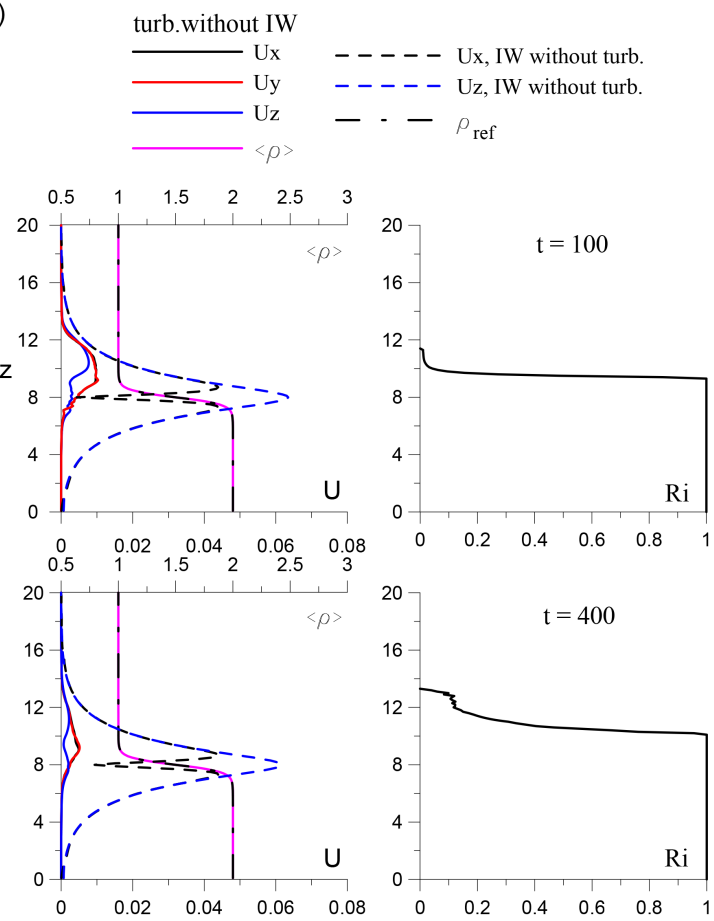

(b)

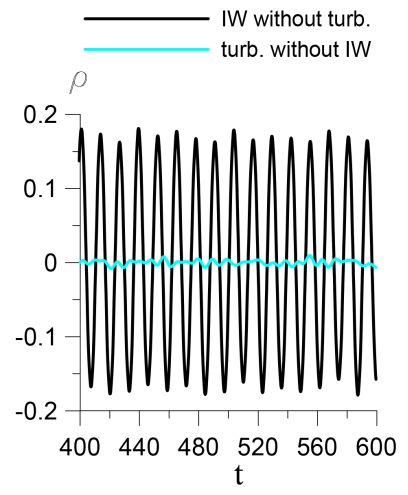

(c)

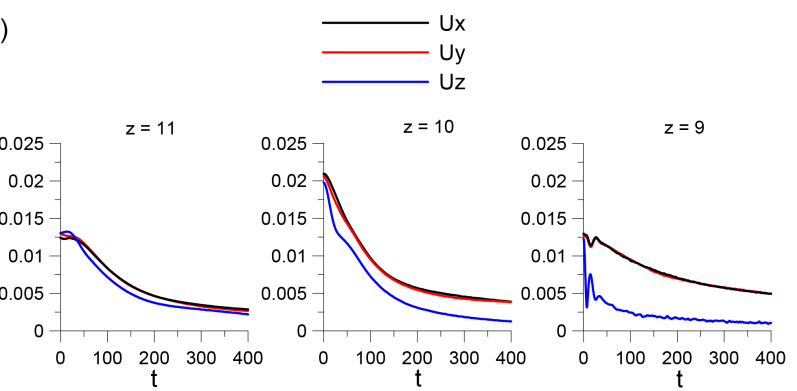

Figure 3. (a) Vertical profiles of the rms velocity components, $U_{x}^{\prime}, U_{y}^{\prime}$ and $U_{z}^{\prime}$, mean density $<\rho>$ (left) and the gradient Richardson number, $R i_{g}$ (right), obtained at time moments $t=100$ and $t=100$ in DNS with no initially induced IW. The reference (initial) density profile, $\rho_{\text {ref }}(z)$, is shown in dash-dotted (black) line for comparison. Profiles of the rms velocity $x$ and $z$ components of IW without turbulence are also shown in dashed line. (b) Temporal development of the instantaneous density deviation at the point with coordinates $x=20, y=10$ and $z=8$ (in the middle of the pycnolcine) obtained in DNS with initially excited IW without turbulence and initially excited turbulence without IW (in color). (c) Temporal development of the rms velocity components $U_{x}^{\prime}, U_{y}^{\prime}$ and $U_{z}^{\prime}$ obtained at different $z$ levels in DNS with no initially induced IW. 
(d)

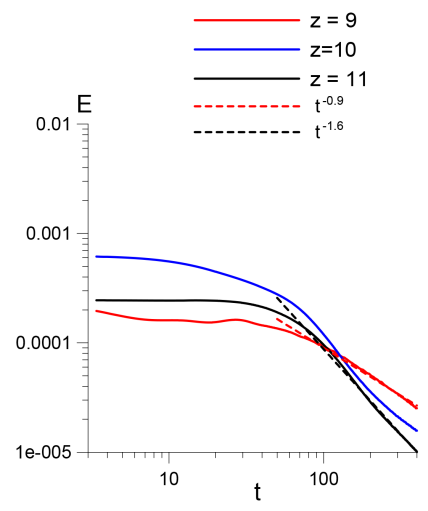

(e)
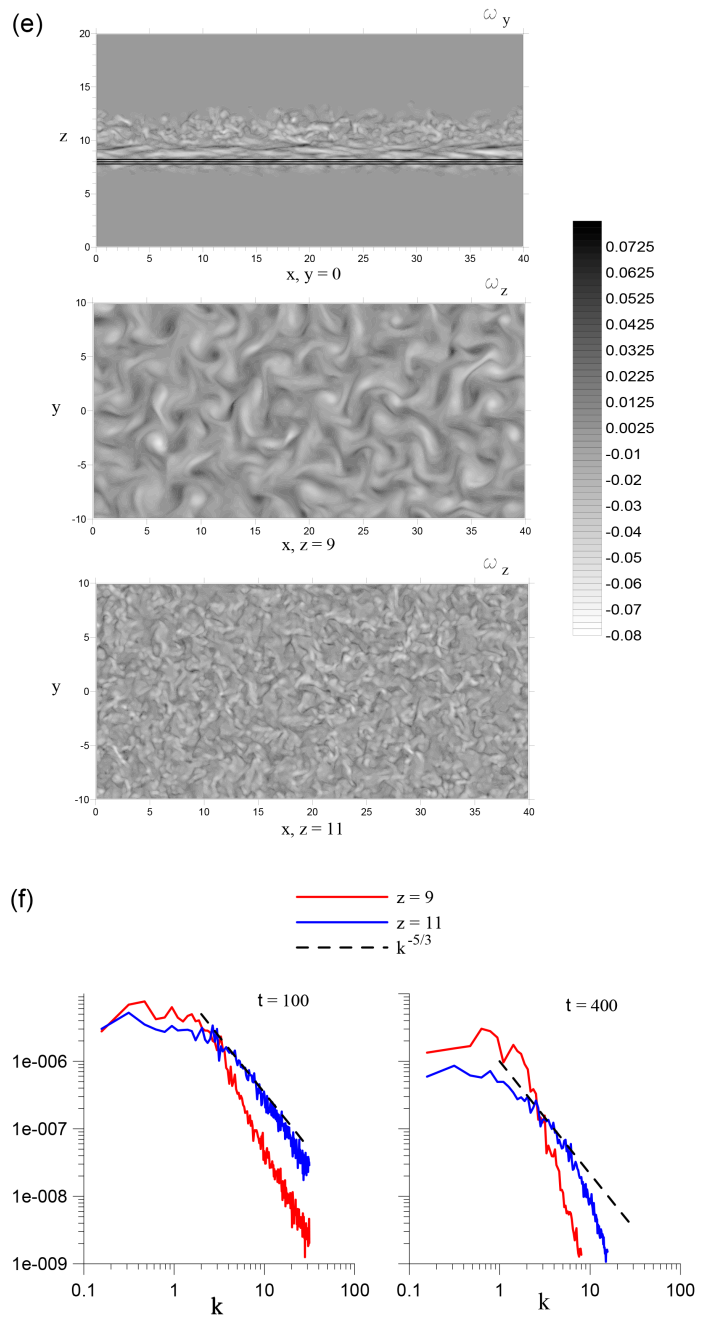

Figure 3. (d) Temporal development of the fluid kinetic energy, $E$, at different $z$ levels $(z=9,10,11)$ in DNS with no initially induced IW. (e) Instantaneous distribution of the vorticity $y$ and $z$ components $\omega_{y}$ (top panel) and $\omega_{z}$ (middle and bottom panels) (in grey scale) obtained in DNS in the vertical and horizontal planes at $t=400$ with no initially excited IW. Density contours $1.3,1.5$ and 1.7 are also shown in the $(x, z)$ plane (top panel). (f) Kinetic energy power spectrum obtained in DNS with no initially excited IWs at $t=100$ (left) and $t=400$ (right) at different $z$ levels. Dashed line shows Kolmogorov's $k^{-5 / 3}$ spectrum. 


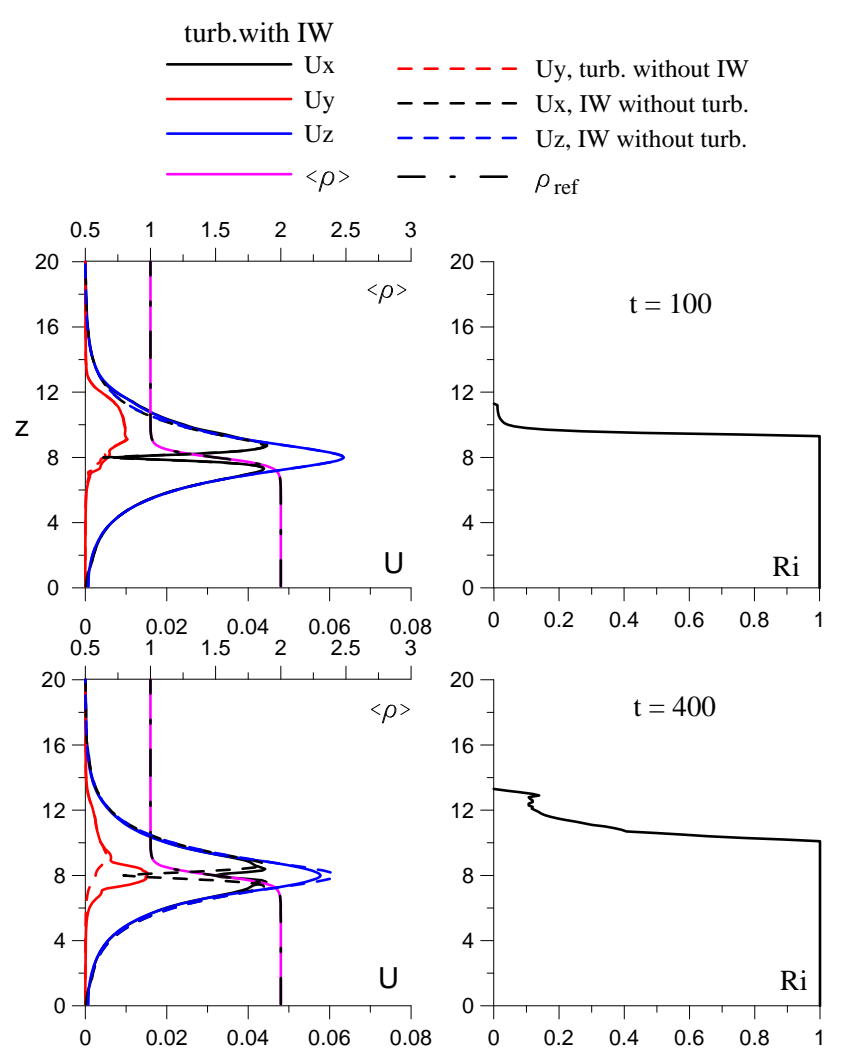

Figure 4. Vertical profiles of the rms velocity components, $U_{x}^{\prime}, U_{y}^{\prime}$, $U_{z}^{\prime}$, mean density $\langle\rho\rangle$ (left) and the gradient Richardson number, $R i_{g}$ (right), obtained at time moments $t=100$ and $t=100$ in DNS with initially induced IW. The reference (initial) density profile, $\rho_{\text {ref }}(z)$, is shown in dash-dotted (black) line. Profiles of the rms velocity $x$ and $z$ components of IW without turbulence are also shown in dashed line for comparison.

Let us consider now the instantaneous distribution of the flow vorticity presented in Fig. 3e. The figure shows $y$ and $z$ components of vorticity, $\left(\omega_{y}=\partial_{z} U_{x}-\partial_{x} U_{z}\right.$ and $\omega_{z}=$ $\left.\partial_{x} U_{y}-\partial_{y} U_{x}\right)$, and density $\left(\rho+\rho_{\text {ref }}(z)\right)$ obtained in DNS in the vertical and horizontal planes with no initially induced IW field at time $t=400$. The figure shows that, sufficiently far from the pycnocline (at $z>10$ ), turbulence remains threedimensional. However, in the vicinity of the pycnocline (in the region $8<z<10$ ), the vorticity distribution in the vertical $(x, z)$ plane is characterized by a layered structure typical of stably stratified turbulence. The scale of vortices in the horizontal $(x, y)$ plane at $z=9$ (where velocities $U_{x}^{\prime}$ and $U_{y}^{\prime}$, and consequently the horizontal kinetic energy, have a maximum) is larger as compared to the $(x, y)$ plane at $z=11$, and turbulent eddies here acquire a pancake shape. This observation is in accord with results of previous laboratory studies where formation of pancake large-scale vortex structures in decaying, strongly stratified homogeneous turbulence was observed (cf. Praud et al., 2005). The figure shows also that strong variability of turbulence in the $z$ direction persists in the strongly stratified region in the vicinity of the pycnocline $(8<z<10)$ and, in this region, $y$ and $z$ vorticity components are generally on the same order.

Figure 3f shows the kinetic energy power spectrum, $E(k)$, obtained in DNS at different $z$ levels $(z=9$ and $z=11)$ at time moments $t=100$ and $t=400$. Each spectrum is obtained by the Fourier transform over the $x$ coordinate at different $y$ locations and then spatially averaged in the $y$ direction. The figure shows that the spectrum obtained sufficiently far from the pycnocline, at $z=11$, is characterized by an inertial interval (for $2<k<20$ at $t=100$, and $2<k<8$ at $t=400$ ), and a viscous dissipation range at larger $k$. On the other hand, the spectrum obtained at $z=9$ is characterized by larger values of the kinetic energy at low wave numbers $(k<3)$ and by faster decay of $E(k)$ at high $k$.

Therefore, the DNS results show, during the considered time interval $(0<t<400)$, that turbulence decay is significantly affected by stratification in the vicinity of the pycnocline, in the region $8<z<11$. In this region, there is a collapse of three-dimensional turbulence and formation of quasi-two-dimensional pancake vortex structures. The horizontal spatial scale of these structures is considerably larger as compared to the characteristic size of three-dimensional turbulent eddies that still survive in the non-stratified region sufficiently far from the pycnocline. In the latter region, the decay rate of the turbulent kinetic energy is enhanced as compared to the region in the vicinity of the pycnocline ( $E(z=11) \sim t^{-1.6}$ as compared to $\left.E(z=9) \sim t^{-0.9}\right)$. As a result, the location of the kinetic energy maximum is shifted with time from the center of the turbulent layer at $z_{\mathrm{t}}=10$ (at $t=0$ ) to the level $z=9$, i.e., closer to the pycnocline. At sufficiently late times ( $>400)$, most of the turbulent kinetic energy is located in a layer occupied by pancake large-scale eddies in the vicinity of the pycnocline.

Figure 3a shows that, during the considered times, the rms turbulence velocity is smaller almost by the order of magnitude as compared to the velocity of initially induced IW without turbulence ( 0.01 vs. 0.06 at $z=9$; cf. Fig. 3a). Thus, the internal wave created by an initial condition $(8-10)$ can indeed be regarded as strong compared to the decaying turbulence. In the next section, we study how this strong internal wave propagating through the pycnocline modifies turbulence dynamics.

\section{Turbulence dynamics in the presence of IWs}

DNS was performed with both the initially created turbulent layer and the internal wave field (Eqs. 8-10) prescribed at $t=0$ with amplitude $W_{0}=0.1$ and wavelength $\lambda=10$. The above presented results show that, for these parameters, the IW rms velocity exceeds turbulence velocity almost by the order of magnitude at sufficiently late times $(t>100)$.

Figure 4 shows vertical profiles of the rms velocities, $U_{x}^{\prime}, U_{y}^{\prime}$ and $U_{z}^{\prime}$, mean density, $<\rho>$ (left panel), and gradi- 
ent Richardson number, $R i_{g}$ (right panel), obtained in DNS at $t=100$ (top) and $t=400$ (bottom). The figure also shows the rms velocity profile $U_{y}^{\prime}$ of turbulence in the absence of IW and the profiles $U_{x}^{\prime}$ and $U_{z}^{\prime}$ due to IW propagating in the pycnocline without initially induced turbulence. The figure shows that, at the considered times, the profiles of $U_{x}^{\prime}$ and $U_{z}^{\prime}$ velocities of IW propagating in the pycnocline with and without initially created turbulence practically coincide. That means that the IW field is weakly affected by turbulence; i.e., the effect of IW damping by turbulence can be regarded as small during the considered time interval. This is in agreement with the results of the previous DNS study by Druzhinin et al. (2013) showing that an IW is effectively damped only if turbulence amplitude is at least twice as large as compared to the IW amplitude.

It is important to note that, in the considered case of turbulence decaying in the presence of IW propagating in the pycnocline, $U_{x}^{\prime}$ and $U_{z}^{\prime}$ velocities include contributions due to both small-scale turbulence and IW fields. Thus, in order to distinguish the effect of IW on turbulence, we compare the profiles of the transverse velocity component, $U_{y}^{\prime}$ (cf. the cases of turbulence with an IW vs. turbulence without an IW in Fig. 4), since this velocity component does not include the contribution due to IW fields (which has only $x$ - and $z$-velocity components). Figure 4 shows that, at early times $(t=100)$, the $U_{y}^{\prime}$ profiles of turbulence, decaying in both the absence of an IW and with an IW, coincide. However, at late times $(t=400)$, velocity $U_{y}^{\prime}$ is considerably enhanced (almost by the order of magnitude) in a layer close to the pycnocline center, at $z \approx 8$, as compared to the $y$ velocity of turbulence without an IW.

Note that generation of small-scale turbulence by internal waves was also observed in the laboratory experiment by Matusov et al. (1989). In that experiment, a small-scale, stationary turbulence layer was created by an oscillating grid at some distance above the pycnocline, whereas an internal gravity wave was simultaneously induced in the pycnocline by a wave maker. The experimental results show that if the forcing by grid was switched off, turbulence decayed in the bulk of the flow domain but survived in a thin layer in the vicinity of the pycnocline center as if maintained by IW. This observation is in qualitative agreement with our results in Fig. 4.

Figure 5a compares the temporal development of rms velocity component $U_{y}^{\prime}$ at the pycnocline center (i.e., at $z=8$ ) obtained in DNS with and without initially induced IW. The figure shows that, under the influence of IW, turbulence kinetic energy increases with time, so that at $t=400, U_{y}^{\prime}$ of turbulence with IW exceeds the velocity of freely decaying turbulence almost by the order of magnitude.

In order to investigate how growing turbulence affects the internal wave, we compare the temporal oscillations of the density deviation in IW with and without turbulence in Fig. 5b. The figure shows that, during the considered time interval, IW is weakly modified by turbulence. As already dis-
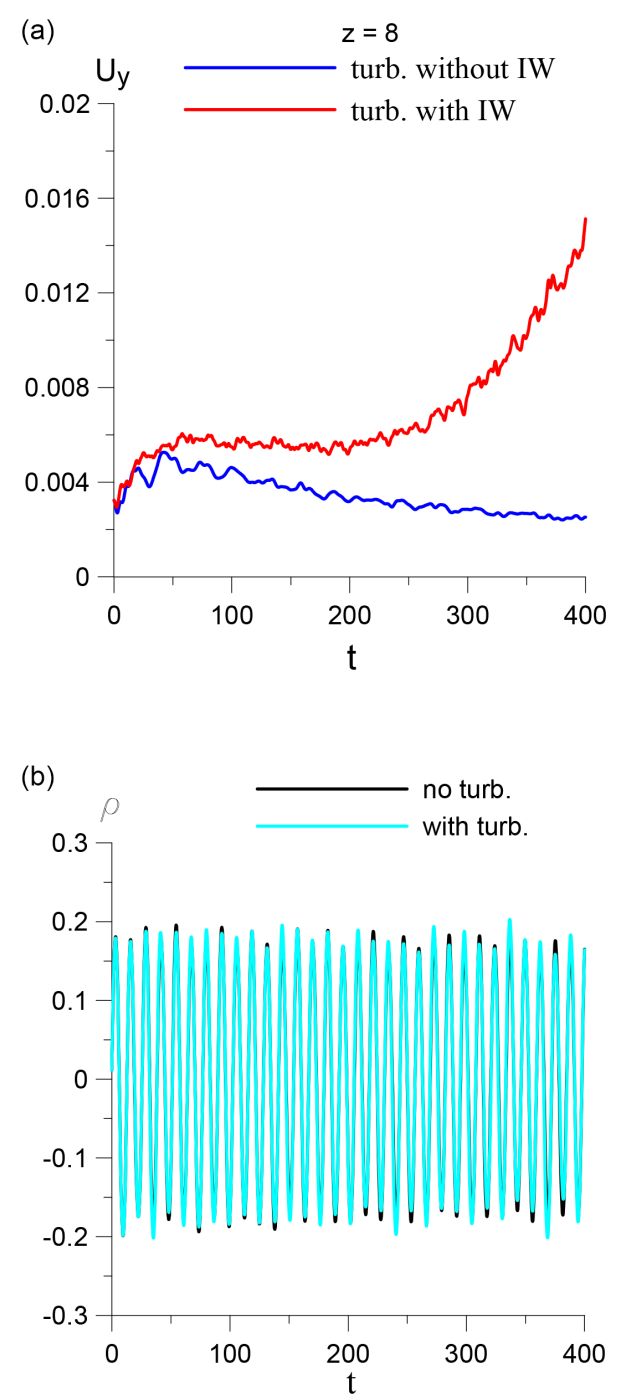

Figure 5. (a) Temporal development of rms velocity component $U_{y}^{\prime}$ obtained at level $z=8$ in DNS with initially excited turbulence and an IW propagating in the pycnocline. Temporal development of turbulence rms velocity in the absence of IW is also shown for comparison. (b) Temporal development of the instantaneous density deviation at the point with coordinates $x=20, y=10$ and $z=8$ (in the middle of the pycnolcine) obtained in DNS with initially excited IW with and without turbulence.

cussed above, this observation agrees with previous results by Druzhinin et al. (2013).

Figure 6 shows instantaneous distributions of the $y$ component of vorticity $\left(\omega_{y}=\partial_{z} U_{x}-\partial_{x} U_{z}\right.$, in grey scale) and density $\left(\rho+\rho_{\text {ref }}(z)\right.$, isolines and grey scale) obtained in DNS of induced turbulence and an IW propagating in the pycnocline at times $t=100$ and 400 . The vorticity distribution in Fig. 6 (top panel) shows that at time $t=100$ there are two distinct regions, $7<z<9$ and $9<z<12$, of weakly and strongly stratified turbulence. In the region $7<z<9$, where $R i_{g}>1$ (cf. Fig. 4), the vorticity distribution is character- 


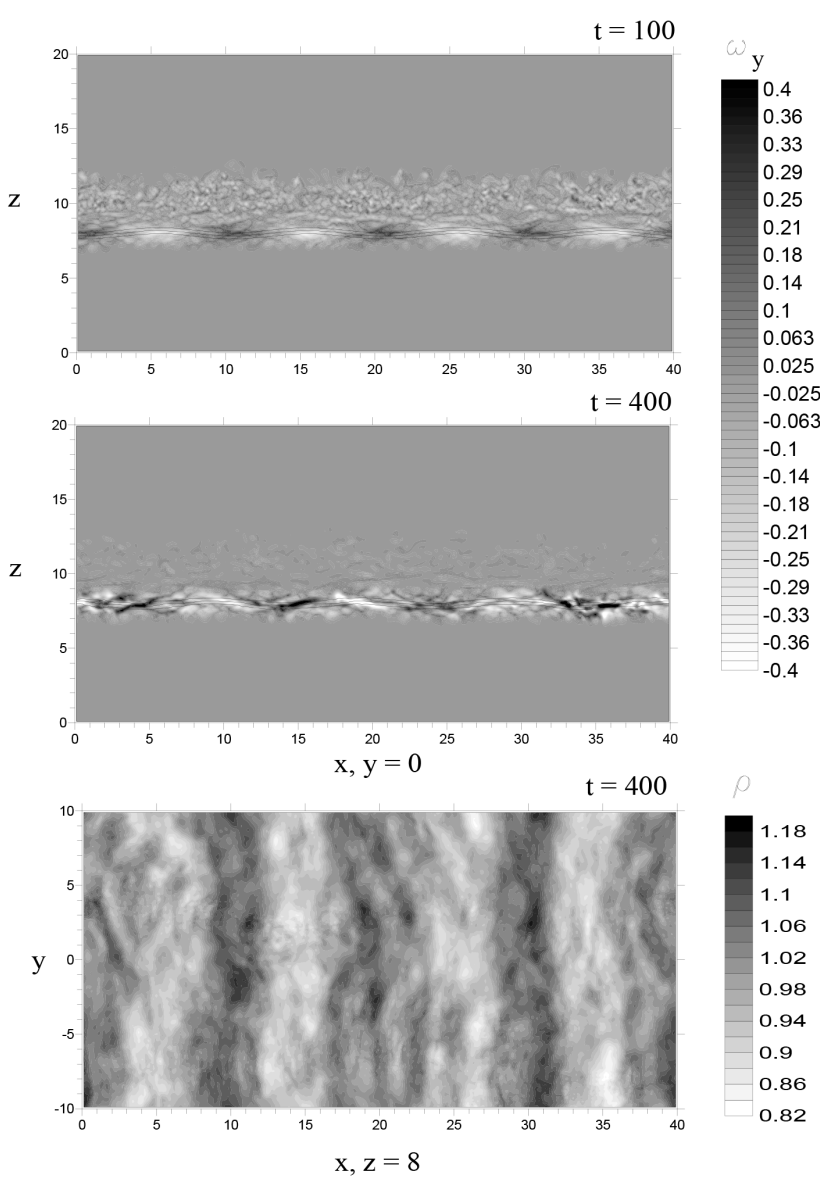

Figure 6. Instantaneous distribution of the vorticity $y$ component $\omega_{y}$ (in grey scale) with imposed density contours $(1.3,1.5,1.7)$ in the central $(x, z)$ plane at time moments $t=100$ and $t=400$ (top and middle panels, respectively), and density distribution in the ( $x$, $y$ ) plane at the pycnocline level $(z=8$, bottom panel) at $t=400$ obtained in DNS of the turbulence layer. IW wavelength $\lambda=10$.

ized by distinct maxima and minima in the vicinity of IW troughs and crests, respectively. In the region $9<z<12$, the Richardson number is small ( $R i_{g}<1$; cf. Fig. 4$)$ and vorticity distribution is similar to that observed in the absence of IW (cf. Fig. 3e). On the other hand, at time $t=400$ (Fig. 7, middle panel), the vorticity is mostly concentrated in the vicinity of the pycnocline, in a thin layer around the pycnocline center at $z \approx 8$. Here, $R i_{g}>1$, and turbulence can be regarded as strongly stratified (cf. Fig. 4). In the upper layer $(z>9)$, the vorticity practically vanishes. Thus, at late times, turbulence is supported by an IW against the effect of the molecular dissipation only in the vicinity of the pycnocline center, and decays in the upper layer. This observation is also in agreement with the laboratory results by Matusov et al. (1989).

It is of interest to note that a similar enhancement of turbulence was observed by Tsai et al. (2015) in the vicinity of the waved water surface. Their DNS results show that turbu-

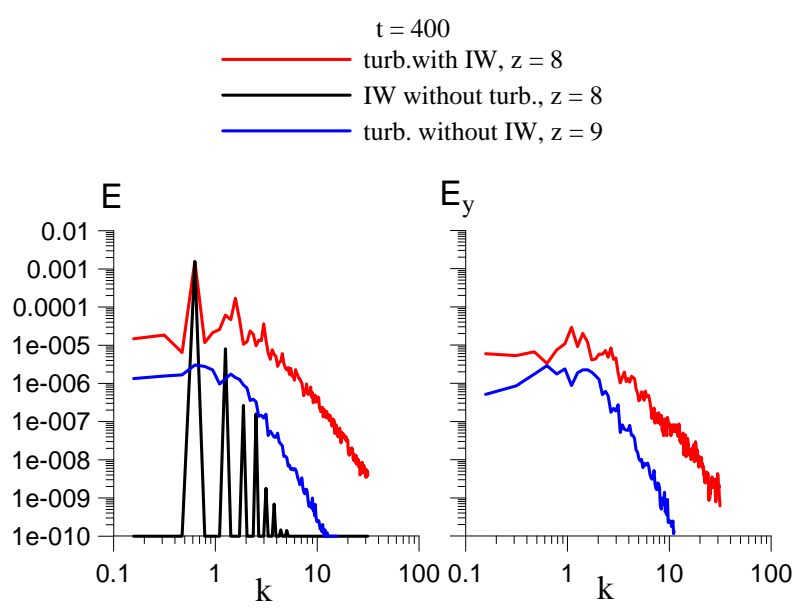

Figure 7. The kinetic energy power spectrum, $E(k)$ (left), and the spectrum of the $y$-velocity component, $E_{y}(k)$ (right), obtained in DNS with initially excited IWs at the pycnocline center level $(z=8)$ at time $t=400$. The kinetic energy spectrum of the internal wave in the absence of a turbulent layer and spectra $E$ and $E_{y}$ of turbulence without an initially induced IW obtained at the level of maximum kinetic energy $(z=9)$ at time $t=400$ are also provided for comparison.

lence is enhanced by the straining field of the surface wave in the vicinity of the water surface, and this enhancement is most pronounced in the vicinity of the surface wave crests and troughs. Since the IW-induced strain field decreases exponentially with the distance from the pycnocline, it is expected that the effect of the IW field on turbulence is most pronounced in the immediate vicinity of the pycnocline, as is observed in our DNS in Fig. 6.

The distribution of the density $\left(\rho+\rho_{\text {ref }}(z)\right)$ at time $t=400$ (Fig. 6, bottom panel) shows that IW is significantly distorted by increased turbulence along the front. This refraction of IW under the effect of turbulence can be the source of more significant IW damping in the case when increasing turbulence amplitude becomes comparable with IW amplitude, as was also observed by Druzhinin et al. (2013).

Figure 7 presents the kinetic energy power spectrum, $E$, and the spectrum of the $y$-velocity component, $E_{y}$, of turbulence with an IW (propagating in the pycnocline) obtained in DNS at the pycnocline center level $(z=8)$ at time $t=400$. The figure also shows the kinetic energy spectrum of the internal wave in the absence of a turbulence layer, and spectra $E$ and $E_{y}$ of turbulence in the absence of an IW at level $z=9$ (where turbulence kinetic energy has a local maximum at $t=400$; cf. Fig. 3a).

Figure 7 shows significant amplification (by the order of magnitude) of the kinetic energy spectrum under the effect of IW in the entire wave-number $(k)$ range. The maximum peak in the IW spectrum (in the absence of turbulence) is due to the first harmonics at $k=2 \pi / 10$, the second-harmonics peak (at $k=4 \pi / 10$ ) being less by 2 orders of magnitude (Fig. 7, 
left panel). That means that the nonlinearity of IW is small and that the internal wave is far from breaking. The kinetic energy spectrum of turbulence with IW is also characterized by a well-pronounced peak at the first-harmonics wave number $(k=2 \pi / 10)$, and the amplitude of this peak practically equals the amplitude of the first-harmonics peak in the IW spectrum without turbulence. That means that, at this wave number $(k=2 \pi / 10)$, the direct contribution of IW to the kinetic energy is most prominent. On the other hand, spectrum $E(k)$ of turbulence with IW is also significantly enhanced (as compared to the turbulence spectrum in the absence of IW) at other $k$, where there is no direct contribution of IW to kinetic energy. Note also that since the energy peak at the IW wave number $k=2 \pi / \lambda=0.628$ in the TKE spectrum is most pronounced, the turbulent length scale, in this case, is actually determined by the IW length $(\lambda=10)$. Then the turbulent Reynolds number can be estimated as $R e_{\mathrm{t}}=L_{\mathrm{t}} U_{\mathrm{t} 0} R e$ $=\lambda U_{\mathrm{t} 0} R e=20000$ for the amplitude $U_{\mathrm{t} 0}=0.1$.

Comparison of the spectra of the $y$-velocity component, $E_{y}(k)$, obtained in DNS with and without IW propagating in the pycnocline (Fig. 7, right panel), shows that the spectra coincide at the wave number of the first IW harmonics $(k=2 \pi / 10)$, and $E_{y}(k)$ of turbulence with IW is significantly enhanced for both lower and higher $k$. Note that since the IW velocity field consists only of $x$ and $z$ components, there is no direct contribution of the IW field in the $E_{y}(k)$ spectrum.

\section{Conclusions}

We have performed DNS of turbulence dynamics in the vicinity of a pycnocline and studied the effect that a monochromatic internal wave propagating along the pycnocline incurs on turbulence dynamics.

DNS results show that, if no IW is initially induced in the pycnocline, turbulence decays and the turbulence kinetic energy (TKE) decreases with time. The TKE decay rate is reduced in the vicinity of the pycnocline. We assume that this reduction of the TKE decay rate can be related to a growing horizontal length scale of turbulent eddies due to the stable stratification effect. At sufficiently late times, most of the turbulent energy is located in a layer close to the pycnocline. Here, the local Richardson number (defined by the local buoyancy frequency and TKE dissipation rate) is large $(R i \gg 1)$, and turbulence dynamics is dominated by quasitwo-dimensional large-scale (pancake) vortex structures.

DNS results also show that, under the effect of an internal wave (IW) propagating in the pycnocline, both the mean kinetic energy of turbulence and the kinetic energy spectrum are significantly enhanced (almost by the order of magnitude) in the vicinity of the pycnocline center as compared to the case of turbulence decaying without initially induced IW. This observation is in qualitative agreement with the results of the laboratory experiment by Matusov et al. (1989).
In conclusion, let us briefly discuss a possible scaling of the above results to typical laboratory and in situ conditions. In the present study, we employ velocity scales, length scales and timescales, $U_{0}, L_{0}$ and $T_{0}=L_{0} / U_{0}$, to normalize physical variables. Note that since the timescale is defined as $T_{0}=1 / N_{0}$ and the velocity scale as $U_{0}=L_{0} / T_{0}=L_{0} N_{0}$, the bulk Richardson number in DNS identically equals unity, $R i=N_{0}^{2} L_{0}^{2} / U_{0}^{2}=1$. For laboratory conditions, we prescribe $L_{0}=20 \mathrm{~cm}$ (IW wavelength $\lambda=2 \mathrm{~m}$, pycnocline thickness $20 \mathrm{~cm}$ ) and for the considered Reynolds number $R e=20000$ and kinematic viscosity $v=0.01 \mathrm{~cm}^{2} \mathrm{~s}^{-1}$, we obtain $U_{0}=\operatorname{Rev} / L_{0}=10 \mathrm{~cm} \mathrm{~s}^{-1}$ for initial turbulence velocity and $0.1 U_{0}=1 \mathrm{~cm} \mathrm{~s}^{-1}$ for IW vertical velocity amplitude; the timescale is $T_{0}=2 \mathrm{~s}$ and the buoyancy frequency is $N_{0}=0.5 \mathrm{rad} \mathrm{s}^{-1}$. Then, extrapolating our results to oceanic conditions, we take $N_{0}=0.01 \mathrm{rad} \mathrm{s}^{-1}$ (Phillips, 1977), i.e., $T_{0}=100 \mathrm{~s}$ for the timescale and $L_{0}=20 \mathrm{~m}$ for the length scale (IW wavelength $200 \mathrm{~m}$, pycnocline thickness $20 \mathrm{~m}$ ). Thus, the velocity scale is $U_{0}=20 \mathrm{~cm} \mathrm{~s}^{-1}$, and initial turbulence velocity and the IW vertical velocity amplitude are both $0.1 U_{0}=2 \mathrm{~cm} \mathrm{~s}^{-1}$. Although the analysis of specific oceanic situations is beyond the framework of this paper, it provides a stricter mathematical confirmation of the early conclusions by Matusov et al. (1989).

Acknowledgements. This work was supported by RFBR (Project Nos. 14-05-00367, 15-05-02430).

Edited by: H. J. Fernando

Reviewed by: I. Esau and S. Sukoriansky

\section{References}

Anis, A. and Moum, J. N.: Surface wave-turbulence interactions: scaling $\varepsilon(z)$ near the sea surface, J. Phys. Oceanogr., 25, 20252045, 1995.

Carruthers, D. J. and Hunt, J. C. R.: Velocity fluctuations near an interface between a turbulent region and a stably stratified layer, J. Fluid Mech., 165, 475-501, 1986.

Druzhinin, O. A., Ostrovsky, L. A., and Zilitinkevich, S. S.: The study of the effect of small-scale turbulence on internal gravity waves propagation in a pycnocline, Nonlin. Processes Geophys., 20, 977-986, doi:10.5194/npg-20-977-2013, 2013.

Fernando, H. J. S.: Turbulent mixing in stratified fluids, Ann. Rev. Fluid Mech., 23, 455-493,1991.

Hazel, P.: Numerical studies of the stability of inviscid stratified shear flows, J. Fluid Mech., 51, 39-61, 1972.

Kantha, L. H.: On generation of internal waves by turbulence in the mixed layer, Dynam. Atmos. Ocean., 3, 39-46, 1979.

Kantha, L. H.: Laboratory experiments on attenuation of internal waves by turbulence in the mixed layer, Trans. 2nd Int. Symp. on Stratified Flows, Irodheim, Norway, IAHB, 731-741, 1980.

Matusov, P. A., Ostrovsky, L. A., and Tsimring, L. S.: Amplification of small scale turbulence by internal waves, Dokl. Akad. Nauk USSR, 307, 979-984, 1989 (in Russian). 
Ostrovsky, L. A. and Zaborskikh, D. V.: Damping of internal gravity waves by small-scale turbulence, J. Phys. Oceanogr., 26, 388397, 1996.

Ostrovsky, L. A., Kazakov, V. I., Matusov, P. A., and Zaborskikh, D. V.: Experimental study of the internal waves damping on smallscale turbulence, J. Phys. Oceanogr., 26, 398-405, 1996.

Phillips, O. M.: The dynamics of the upper ocean, 2nd Edn., Cambridge University Press, Cambridge, 1977.

Praud, O., Fincham, A. M., and Sommeria, J.: Decaying grid turbulence in a strongly stratified fluid, J. Fluid Mech., 522, 1-33, 2005.
Thorpe, S. A.: An introduction to ocean turbulence, Cambridge University Press, Cambridge, 2007.

Tsai, W., Chen, S., and Lu, G.: Numerical evidence of turbulence generated by non-breaking surface waves, J. Phys. Oceanogr., 45, 174-180, 2015

Turner, J. S.: Buoyancy effects in fluids, Cambridge Univ. Press, 1973.

Warhaft, Z. and Lumley, J. L.: Experimental study of decay temperature fluctuations in grid generated turbulence, J. Fluid Mech., $88,659-684,1978$. 